\title{
Lung epithelial endoplasmic reticulum and mitochondrial 3D ultrastructure: a new frontier in lung diseases
}

\author{
Sierra R. Bruno ${ }^{1} \cdot$ Vikas Anathy $^{1}[0$ \\ Accepted: 24 November 2020 / Published online: 18 February 2021 \\ c) Springer-Verlag GmbH Germany, part of Springer Nature 2021
}

\begin{abstract}
It has long been appreciated that the endoplasmic reticulum (ER) and mitochondria, organelles important for regular cell function and survival, also play key roles in pathogenesis of various lung diseases, including asthma, fibrosis, and infections. Alterations in processes regulated within these organelles, including but not limited to protein folding in the ER and oxidative phosphorylation in the mitochondria, are important in disease pathogenesis. In recent years it has also become increasingly apparent that organelle structure dictates function. It is now clear that organelles must maintain precise organization and localization for proper function. Newer microscopy capabilities have allowed the scientific community to reveal, via 3D imaging, that the structure of these organelles and their interactions with each other are a main component of regulating function and, therefore, effects on the disease state. In this review, we will examine how 3D imaging through techniques could allow advancements in knowledge of how the ER and mitochondria function and the roles they may play in lung epithelia in progression of lung disease.
\end{abstract}

Keywords Endoplasmic reticulum $\cdot$ Mitochondria $\cdot$ Lung $\cdot$ Epithelial $\cdot$ Structure $\cdot 3 \mathrm{D}$

\section{Introduction}

Organelles serve as the housing of unique niches within a cell and specialize in various regulatory processes, all essential for proper functioning and survival of the cell. Because these entities maintain cellular homeostasis, dysregulation of organelle function can have dire effects on a cell and, in turn, the organism as a whole. Two organelles known to play significant roles in cellular homeostasis are the ER and mitochondria. The ER is the key regulator of protein folding, post-translational modifications, calcium homeostasis, and lipid synthesis (Braakman and Hebert 2013; Clapham 2007; Fagone and Jackowski 2009; Hebert et al. 2005). Mitochondria are known for their roles in oxidative phosphorylation (OXPHOS), reactive oxygen species (ROS) production, fatty acid oxidation, and calcium flux regulation (Annesley and Fisher 2019; Picard et al. 2011). Dysfunction in these processes in either of the organelles are known to

Vikas Anathy

Vikas.anathy@med.uvm.edu

1 Department of Pathology and Laboratory Medicine, University of Vermont, Larner College of Medicine, 149 Beaumont Ave, Burlington, VT 05405, USA have an impact on overall cell health, leading to various disease states.

Our knowledge of the structure of mitochondria and the ER has improved greatly since their discovery in 1857 and 1945, respectively. The ER is the largest organelle in a cell and is comprised of a continuous membrane structure extending from the nucleus, making up the nuclear envelope, to the periphery of the cell, where it forms sheet- and tubule-like structures (Schwarz and Blower 2016; Westrate et al. 2015). Mitochondria are a double membrane organelle that can be either elongated or punctate and often form an interlaced branched network throughout the cell (Dudkina et al. 2010; Herst et al. 2017; Picard et al. 2011). While a lot of information about each of these organelles has been obtained through 2-dimensional microscopy techniques, more has been and remains to be gained through 3-dimensional microscopy about organization, distribution throughout the cell, and dynamic interactions with other organelles.

One of the organ systems known to be highly impacted by dysfunction of either the ER or the mitochondria is the lungs. A wide variety of lung diseases, including asthma, fibrosis, and response to infection, are regulated by these organelles. Within each of these disease states, various cell types can be seen to play a role in disease progression 
through dysregulation of their ER and mitochondria. However, as one of the most common sites of disease initiation in the lung, epithelial organelle function is of particular importance. In this review, we will attempt how 3-dimensional (3D) imaging techniques have advanced and can further advance our understanding of how airway epithelial ER and mitochondria contribute to disease pathogenesis of various lung diseases.

\section{A 3D view of mitochondria and the ER}

While the overall general structure and function of mitochondria and the ER have been appreciated for quite some time, what has only begun to be appreciated within the last decade or so is the role that the structures of these multifaceted organelles and their dynamic interaction with other organelles within the cell play in dictating proper, or dysregulated, functions. Advances in microscopy technique and analysis have allowed a more comprehensive $3 \mathrm{D}$ view of these organelles and their interactions to advance our understanding of their function in both normal and disease states (Abrisch et al. 2020) (Fig. 1). Here we will discuss the knowledge gained through 3D microscopy techniques of the ER and mitochondria.

The mammalian ER is the largest of the organelles and forms two connected but unique sub-compartments; the nuclear envelope (NE), which encompasses the nucleus, and the peripheral ER, which extends throughout the cytoplasm of the cell. While the NE allows for protection of nuclear material and selective entry and exit for the nuclear space, the peripheral ER is important for calcium regulation, lipid synthesis, and protein folding and modification. Though these sub-compartments are distinct, their shape and proper function are often dictated by the same or similar proteins, and each other. Various 3D microscopy techniques allowing more accurate and complete visualization of this dynamic organelle system have shown that the peripheral ER and NE are drastically altered during cell cycle progression and cell division. During initiation of mitosis, the NE dissipates into the peripheral ER, becoming more tubule-like. Following proper splitting of the nuclear DNA and progression into the late phases of cell division, restructuring of ER cisternae allow the reformation of the NE and redistribution of the peripheral ER in the resulting cells (Anderson and Hetzer 2008; Lu and Kirchhausen 2012; Puhka et al. 2007). Additionally, reticulons and several other proteins, as well as lipids such as diacylglycerol, appear to be key regulators of ER curvature and structural maintenance (Craene et al. 2006; Domart et al. 2012; Lu and Kirchhausen 2012; Ulloa et al. 2019; West et al. 2011a). 3D electron microscopy has also revealed that actin filaments assist in maintenance and stabilization of cisternae of the ER as well (Joensuua et al. 2014). Advancements in microscopy have also allowed an appreciation that the shapes of the various regions of the ER are probably important in dictating proper function of those regions. This is apparent due to the differences in protein localization and organization of the different regions of the ER. The peripheral ER is split into two distinct compartments; the cisternae, or sheet-like, regions and the tubulelike regions. The cisternae, a flat sheet-like region of the ER with low curvature, are thought to be better suited to ER luminal processes such as protein folding due to the larger continuous luminal area and the localization of protein synthesis and folding machinery to these regions of the ER, while ER tubules, which have high amounts of curvature, have a smaller luminal area when compared to surface area and have a decreased amount of protein folding machinery localized to them (Shibata et al. 2006; Voeltz et al. 2002; West et al. 2011b). The control of ER shape and the roles that regulations of shape play in proper function of the ER have further been examined in several detailed reviews (Friedman and Voeltz 2011; Hetzer et al. 2005; Joensuua and Jokitalo 2015; Schwarz and Blower 2016; Westrate et al. 2015).

With an understanding of shape of the ER and how shape dictates function, comes the understanding that dysregulation of ER shape can impact the state of the cell and promote disease. In fact, viral interaction with and impact on the shape of the ER of host cells has already begun to be appreciated. In particular, the flavivirus family of viruses (e.g. Zika Virus, West Nile, Tick-borne Encephalitis Virus) tends to induce vesicle formation. The use of $3 \mathrm{D}$ electron microscopy showed these viral-induced vesicles are formed
Fig. 1 Endoplasmic Reticulum membrane contact sites (green) with inner mitochondrial membrane (magenta). Reproduced from Abrisch et al. (2020)
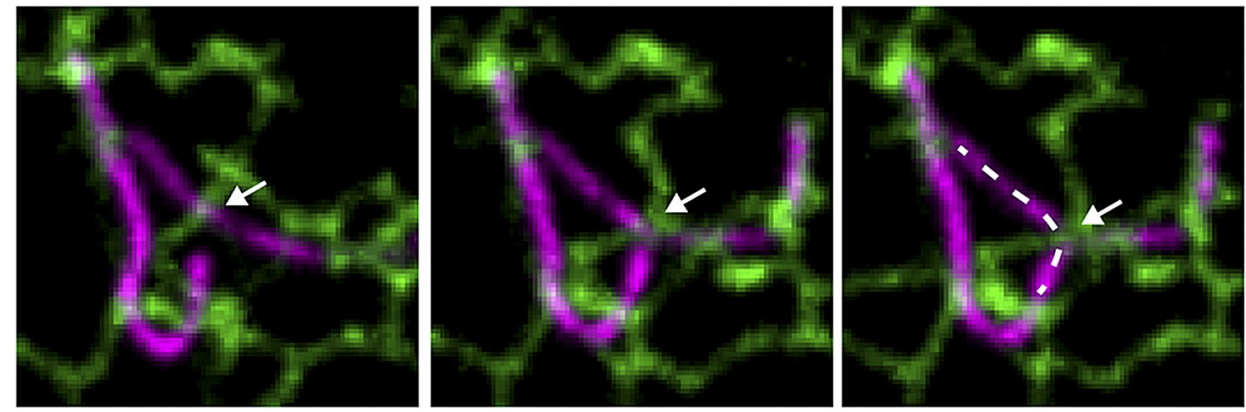
in and are localized to remodeled areas of the ER during infection by various flavivirus members in their target cell types (Bílý et al. 2015; Gillespie et al. 2010; Offerdahl et al. 2012; Stefanik et al. 2018). Flavivirus infection enhances proliferation of the ER to allow viral replication and altered ER cisternae structure where viral vesicles localized (Bílý et al. 2015; Gillespie et al. 2010; Offerdahl et al. 2012; Stefanik et al. 2018). Hepatitis $C$ infection also relies heavily on the ER. 3D electron microscopy and soft X-ray cryo-tomography revealed extensive modification of the ER following viral infection to support viral replication and budding of viral particles from the ER at sites localized closely to lipid droplets within the cell (Pérez-Berná et al. 2016; Roingeard et al. 2008). These results provide substantial evidence to support change in shape of the ER to propagate infection and lends to the possibility of ER alterations propagating other disease states.

Mitochondria are unique organelles in that they have two lipid bilayers, the outer mitochondrial membrane (OMM) and the inner mitochondrial membrane (IMM) forming two distinct luminal spaces. This allows the mitochondria to be diverse in their jobs, as they can set up isolated gradients to generate signaling molecules like ATP and ROS, as well as storage of $\mathrm{Ca}^{2+}$. This dynamic organelle is also responsible for regulation of cell cycle progression and apoptosis (Annesley and Fisher 2019; Chakrabarty et al. 2018; Fakouri et al. 2019; Picard et al. 2011). Microscopy advances and $3 \mathrm{D}$ imaging have made it apparent that these organelles can form long, complex networks or small, punctate structures. In fact, the physical size and branching of mitochondria are very important in dictating function and maintenance of the organelle. The size and organization of mitochondria are tightly controlled through fission and fusion (Bliek et al. 2013; Lee and Yoon 2016). These processes are carried out by dynamin-like GTPases; Drp1 is the main driver of mitochondrial fission while Mfn regulates fusion of the OMM and OPA1 regulates IMM fusion (Lee and Yoon 2016). Studies have indicated that punctate mitochondria perform different functions than elongated mitochondria (Liesa and Shirihai 2013; Yamaguchi et al. 2012). While these structural changes are essential for proper function of cells, they can be altered and are often seen in different configurations and become more specialized depending on the cell type. Muscle cells, for example, form large mitochondrial networks for rapid, coordinated energy production and can be quickly altered if necessary to maintain a healthy, properly functioning mitochondrial network, as visualized using 3D reconstruction of confocal and electron microscopy images (Dahl et al. 2015; Glancy et al. 2017). Neurons, however, have specialized mitochondria based on the sub-compartment of the cell they are localized in. 3D reconstruction after various electron microscopy techniques has shown axonal and pre-synaptic mitochondria are smaller in size, whereas mitochondria localized to the post-synaptic dendrites are longer and more networked (Delgado et al. 2019; Popov et al. 2005). Additionally, 3D confocal and electron microscopy have demonstrated that microtubules are necessary for proper trafficking of mitochondria through the axon to the pre-synaptic space to allow proper mitochondrial localization in bipolar interneurons (Graffe et al. 2015). Functional information can also be gained by understanding structure of this organelle along with protein expression on its surface. Taste receptor cells have expression of CALHM1 in larger mitochondria with atypical cristae located in close proximity to the membrane in the pre-synapse but not on smaller or more typical mitochondria. This channel allows the release of ATP from the mitochondria to act as a neurotransmitter, important for taste cell signaling to the taste neurons (Romanov et al. 2019). Further understanding of protein expression impact on mitochondrial structure was determined in yeast. 3D electron microscopy was used to show that Cdc48p, a highly conserved ATPase also expressed in mammalian cells, is necessary for elongation of mitochondria (Miyazaki et al. 2014). Additional examination of mitochondrial shape and function and visualization of this organelle can be seen in existing reviews (Glancy 2020; Picard et al. 2011).

As with the ER, dysfunction of mitochondria has been linked to numerous diseases (Annesley and Fisher 2019; Chakrabarty et al. 2018; Fakouri et al. 2019; Herst et al. 2017). However, until recent years, the 3D structural alterations of mitochondria during disease were poorly understood. One area that advancements have been made in mitochondrial structural change through use of 3D microscopy techniques is in neuronal diseases. Improper regulation of mitochondrial dynamics through dysfunctional fission and fusion processes have been appreciated in a wide host of neuropathies including aging, Parkinson's, ischemia, and neuroinflammation (Eustaquioa et al. 2018; Katoh et al. 2017; Morozov et al. 2017; Owens et al. 2015). Additionally, an Alzheimer's model in mice was shown to alter mitochondrial cisternae and membrane potential and was linked with loss of antioxidant Peroxiredoxin 3 in hippocampal neurites (Choi et al. 2014). Drug toxicity can also impact mitochondrial structure and function. Ketamine treatment was shown to alter fission and fusion dynamics leading to smaller, swollen mitochondria in developing brains of rats and was linked to increase in apoptosis (Eustaquioa et al. 2018). Another organ system impacted by 3D structural changes is the kidneys. Over-activation of 5'-AMP activated kinase, which is often a method for treatment of various kidney diseases, in renal proximal tubule cells prompted reorganization and fragmentation of mitochondria as visualized by 3D reconstruction of confocal microscopy images (Kodiha et al. 2018). Another treatment, the antioxidant coenzyme $Q_{10}$, was also tested to treat tacrolimus-induced nephrotoxicity. 3D electron microscopy techniques showed that this treatment 
recovered mitochondrial size alterations, along with functional alterations, done to the kidneys by tacrolimus (Yu et al. 2019). Hepatitis C infection has also been shown to alter 3D structure as seen by differences in cristae and mitochondrial distribution (Pérez-Berná et al. 2016). Mitochondrial alterations are also known to cause diseases coined mitochondrial myopathies. 3D electron microscopy techniques used on biopsies of patients with mitochondrial DNA mutations demonstrated alterations in cristae and overall mitochondrial shape and organization (Vincent et al. 2016). All of this data combined with the knowledge that mitochondrial functional alterations are important in disease pathogenesis provides strong evidence that mitochondrial morphology plays an important role in a host of various diseases, of which we are likely just scratching the surface.

While the ER and mitochondria are structurally and functionally important in health and disease on their own, a field of gaining interest is the role of sites of interaction between these two organelles in normal cell function and progression of disease (Csordás et al. 2018; Filadi et al. 2017; Ilacqua et al. 2017; Rowland and Voeltz 2012). 3D microscopy advancements have allowed us to begin to gain new insight into ER-mitochondrial contact sites and their potential roles in disease. 3D reconstruction of soft x-ray tomography and confocal microscopy images revealed that MiD51, a protein known to be involved in mitochondrial fission, localizes to ER-mitochondrial contact sites where the ER forms around the mitochondria, supporting the role of the ER and MiD51 in mitochondrial fission (Elgass et al. 2015). Live cell Z-series confocal images in yeast also showed that ER-mitochondrial contact sites not only control fission dynamics, but fusion as well, indicating a key role for the ER in dynamic mitochondrial regulation (Abrisch et al. 2020). Another 3D microscopy study determined that ER-mitochondrial contact sites, which are important for calcium transport between the two organelles in order to regulate cellular calcium levels, are not decreased with increases in mitochondrial fission, but that calcium transfer between the two organelles is decreased and protects the cell from apoptosis (Szabadkai et al. 2004). The dynamics of calcium transport between these two organelles was further examined when adenine nucleotide translocase, a protein normally responsible for ATP transfer out of the mitochondria. Overexpression of this mitochondrial protein decreased mitochondrial uptake of calcium from the ER and induced mitochondrial fission, but did not impact the number of ERmitochondrial contact sites as seen by 3D confocal microscopy (Wieckowski et al. 2006). These 3D microscopy studies taken together are opening up and furthering our understanding of how these organelles interact with and impact each other through alterations of their own biology and how this may impact disease states.

\section{Airway epithelial ER and mitochondria are key drivers of lung disease pathogenesis}

Lung epithelial cells are now appreciated as main contributors to initiation and/or progression of a wide host of lung diseases. As the first line of defense in the lung, epithelial cells are continuously exposed to inhalants of a large variety, including such things as allergens, viruses, and pollutants. In turn, airway epithelia can become injured or stimulated and can induce signaling cascades to promote disease pathogenesis. The ER and mitochondria have been previously proven to play integral roles in the epithelial response to these various stimuli and in regulation of the disease state. Here, we will examine the impact these organelles have on the airway epithelial response to various lung diseases.

A major functional role of the ER is the folding of proteins to be integrated into cellular membranes or secreted from the cell. If, for any reason, the cell produces proteins that cannot be folded properly or produces an overwhelming amount of proteins that need to be folded, ER stress will be induced and the unfolded protein response (UPR) will be activated. This response, propagated by activation of the three canonical ER-membrane bound transducers of UPR signal, ATF6, IRE1, and PERK, will induce a signaling cascade within the cell to reduce overall transcription and translation and promote chaperone activity to ease the burden on the ER or push the cell into apoptosis if it cannot resolve the stress (Hetz 2012; Hetz et al. 2020). The epithelial UPR is known to play a role in progression of a wide range of diseases in the lung. RNA-sequencing of asthmatic epithelial brushings has demonstrated that asthmatics have increased transcription of ER stress markers as well as increased alternative splicing of XBP1 induced by IRE1 activation, resulting in translation of a transcription factor that upregulates expression of proteins to help resolve the ER stress (Bhakta et al. 2018). In addition, epithelial expression of the chaperone and protein disulfide isomerase ERp57 along with ER stress receptors ATF6 $\alpha$ and IRE1 $\beta$ were shown important for pathogenesis of asthma (Hoffman et al. 2013, 2016; Martino et al. 2013). The role of epithelial ER stress in asthma pathogenesis was further corroborated by a study demonstrating that allergen-specific immunotherapy targeted to treatment of $D e r f$-induced asthma ameliorated the asthmatic response by reducing the IL-25-induced ER stress response and epithelial apoptosis (Yuan et al. 2018). UPR pathways are also activated in and suggested to play a key role in disease establishment and progression of pulmonary fibrosis in both patients with and models of pulmonary fibrosis (Katzen et al. 2019; Korfei et al. 2008; Lawson et al. 2008, 2011; Tanjore et al. 2015; Torres-González 
et al. 2012; Zhong et al. 2011). Supporting these data, inhibition of various UPR markers blocked development of or reversed fibrosis phenotype in models of the disease (Burman et al. 2018; Delbrel et al. 2018; Thamsen et al. 2019; Zheng et al. 2019). Additionally, overexpression of or enhanced activity of UPR markers worsened the fibrotic phenotype (Chen et al. 2019; Lawson et al. 2011). Pulmonary viral infections also initiate and rely on the UPR. Several Human Coronaviruses upregulate UPR activation (Siu et al. 2014) as does Human Rhinovirus 16 (Song et al. 2019). Influenza A Virus (IAV) upregulates various UPR proteins including IRE1, ATF6, and ERp57, which assists in folding of viral proteins, cell survival, and viral propagation (Chamberlain et al. 2019; Hassan et al. 2012; Roberson et al. 2012). Interestingly, PERK pathway activation appears to reduce IAV replication, as seen with use of the IAV inhibitor Montelukast (Landeras-Bueno et al. 2016). Respiratory Syncytial Virus (RSV) infection also activates the IRE1 and ATF6 pathways of the UPR and IRE1 activation, in particular, is important for RSV replication (Hassan et al. 2014). ER membrane fluidity and permeability also play a role in allergic airway inflammation (Lee et al. 2020).

Mitochondria, as a multifaceted organelle, play an integral role in airway epithelia function. As airway epithelia are often important for initiation of disease responses, their mitochondrial needs and outputs would presumably need to change to meet the new needs of the cell. Multiple mitochondrial pathways could be impacted in the same disease state within the epithelia. In fact, several physical and functional changes in mitochondria in airway epithelia are linked to numerous lung diseases. Asthmatic patients have been shown to have altered bronchial epithelia mitochondrial ultrastructure (Konrádová et al. 1985). An ovalbumin mouse model of asthma has also shown alterations in bronchial epithelial cell mitochondrial size and cristae, along with altered mitochondrial respiration and reduced ATP production, linked to increased IL4 levels in the lung (Mabalirajan et al. 2008). Similarly, ragweed exposed cultured airway epithelia and a ragweed-induced mouse model of asthma demonstrated oxidative damage to subunit II of the ubiquinol cytochrome c reductase promoted increased mitochondrial reactive oxygen species and was linked to increased airway hyper responsiveness, eosinophilia, and mucus metaplasia (AguileraAguirre et al. 2009). Inhibition of mitochondrial reactive oxygen species produced during the asthmatic response, in both cultured epithelia and in ovalbumin challenged mice, reduced NLRP3 inflammasome activation and, in the mouse model, decreased several hallmarks of asthma including inflammation and airway hyper responsiveness (Kim et al. 2014). Mitochondrial arginine metabolism has also been shown to be important in the asthmatic response

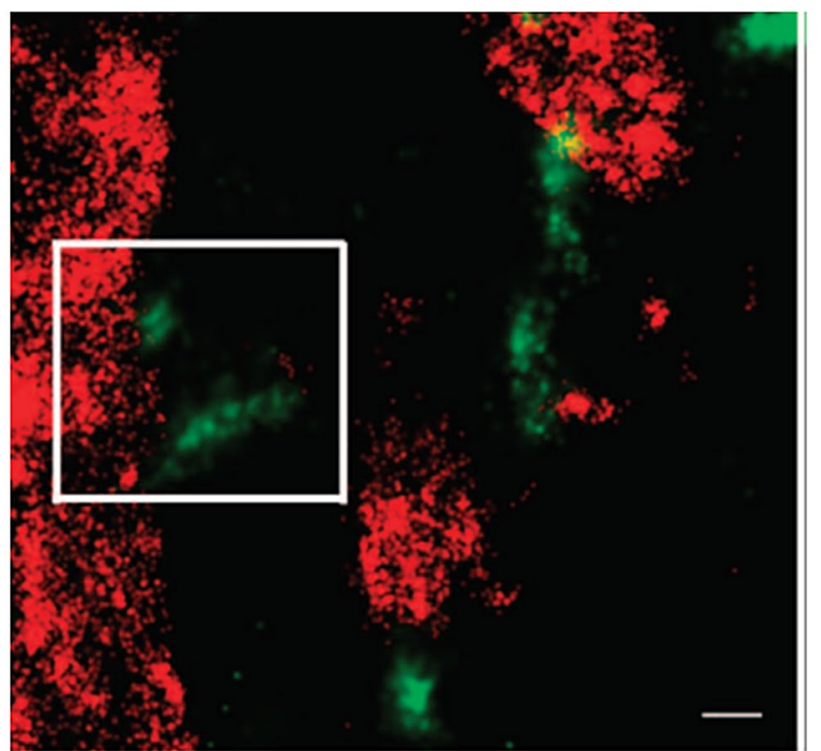

Fig. 2 Accumulation of influenza viral NP on the mitochondria of lung epithelial cells by STORM microscopy and 3D reconstruction (red-TOM20, Green-Influenza Viral NP). Reproduced from Liedmann et al. (2014)

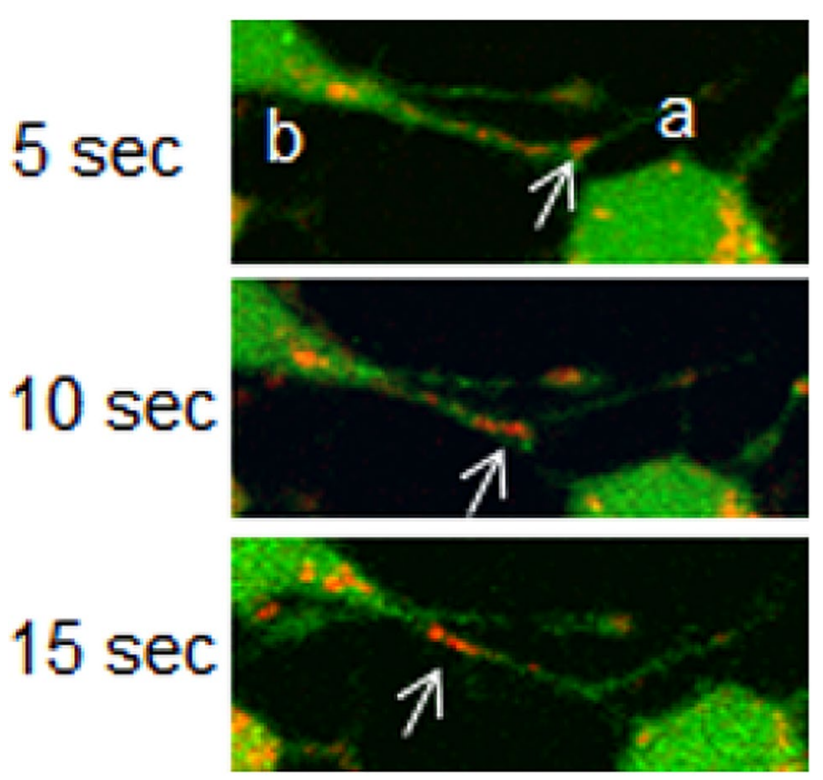

Fig. 3 Confocal images show transfer of mesenchymal bone marrow stem cell mitochondria to epithelial cells during LPS induced lung injury (red, arrows). Reproduced from Islam et al. (2012)

to allergen, with arginase- 2 increases, which are also seen in asthmatic patient airways, helping suppress the inflammatory phenotype developed in asthma (Xu et al. 2016). Rhinosinusitis has been linked to mitochondrial dysfunction as well. Both cultured epithelia exposed to Staphylococcus aureus enterotoxin $\mathrm{B}$, a common pathogen seen in 
Rhinosinusitis patients, as well as epithelial nasal polyps from Rhinosinusitis patients exhibited altered oxidative phosphorylation, reactive oxygen species production, and mitochondrial structural dynamics (Yoon et al. 2020). Pulmonary fibrosis is considered a disease of age. Aged rat lungs exhibit oxidative damage and stress, NAD + / NADH ratio decrease, and decreased mitochondrial respiration (Braidy et al. 2011). Aged mice, pulmonary fibrosis models, and samples from pulmonary fibrosis patients decreased expression of mitochondrial antioxidant SIRT3 (Sosulski et al. 2017). Several other studies of pulmonary fibrosis also demonstrated in patient and cell culture or mouse model samples that lung epithelial cells in a pro-fibrotic setting exhibit mitochondrial damage and decreased mitophagy, making them more susceptible to damage and promotion of fibrosis (Bueno et al. 2015; Hawkins et al. 2015; Patel et al. 2015). Viral infection of airway epithelia can also alter mitochondrial function. H9N2, an avian Influenza A strain, has been shown to promote cytochrome $\mathrm{c}$ release from epithelial mitochondria and induce apoptosis (Xing et al. 2011). The H9N2 pathogenicity factor PB1-F2, in particular, interacts with mitochondrial localized apoptosis inhibitor HAX-1, enhancing epithelial apoptosis (Li et al. 2018). H5N1, another Influenza A strain, also enhances cytochrome c release from epithelial mitochondria, promoting apoptosis (Bian et al. 2017). One mechanism by which this strain might induce apoptosis is by reducing expression of the epithelial superoxide dismutase SOD, which when present reduces mitochondrial reactive oxygen species, enhances mitochondrial membrane potential, and protects against apoptosis (Lin et al. 2016). Respiratory syncytial virus also impacts lung epithelial mitochondria by enhancing perinuclear clustering of the mitochondria, decreasing mitochondrial membrane potential and respiration, and increasing mitochondrial reactive oxygen species production, which enhances viral propagation (Hu et al. 2019).

\section{D imaging of epithelial ER and mitochondria in lung disease-area for advancement}

While the ER and mitochondria are large contributors to airway epithelial responses during lung disease, and while we know that the structure of these organelles are important in dictating function, little has been done to advance our understanding of the 3D structures of these organelles in this context. Little to no papers exist on 3D ER structure or 3D dynamics of ER-mitochondrial interaction in airway epithelia during lung disease initiation or pathogenesis. And the 3D structure or mitochondria in these cells during different lung diseases has only just begun to be understood. One lung disease environment where 3D organization of mitochondria has begun to be explored is viral infection. As mitochondria play an important role during lung epithelial infection by $\mathrm{RSV}$, one group used various 3D fluorescence microscopy techniques to show localization of RSV Non-Structural protein 1 (NS1) to the mitochondria through direct interaction with Mitochondrial Antiviral Signaling protein (MAVS). NS1 interaction to MAVS inhibits MAVS binding to Retinoic acid Inducible Gene 1 (RIG-1), which is necessary for induction of the IFN inflammatory signaling response by airway epithelia (Boyapalle et al. 2012). Mitochondrial regulation of IFN inflammatory signaling though RIG-1 activation can also play a role during airway epithelial infection by Influenza viruses. As such, 3D STORM microscopy has been used in combination with other techniques to examine the interaction of Influenza Viral Ribonucleoproteins (vRNPs) with the mitochondria of target epithelia and the role this plays in IFN signal regulation. While this study revealed vRNPs interact with the mitochondria of infected cells, and this may induce the IFN viral response while other viral proteins aim to inhibit the response, suggesting a complex nature to mitochondrial interaction with viral proteins (Liedmann et al. 2014) (Fig. 2). Furthermore, 3D imaging studies showed that injured lung alveolar epithelium is repaired by the donation of healthy mitochondria from the mesenchymal bone marrow derived cells (Islam et al. 2012) (Fig. 3). These data suggest that understanding mitochondrial 3D organization and interaction with other components within airway epithelia could prove important in understanding the initiation and progression of various lung diseases including viral infection.

\section{Final thoughts}

Advancements in microscopy have opened the door to examination of the 3D structures and organization of organelles and their interactions with other cellular components. With this progression of techniques to visualize cellular components, we have gained an understanding that the location and structure of the ER and mitochondria are important in dictating function. And while we know that ER and mitochondrial functional alterations in airway epithelia are important for pathogenesis of various lung diseases, what remains to be elucidated is the role that structural and organizational alterations of these organelles might play in such diseases. Filling this gap in knowledge could provide useful in understanding the complex regulation of lung disease states by the ER and mitochondria and open new avenues for treatment. 
Funding This work is supported by NIH R01s HL122383, HL136917, and HL141364 to VA, and by a NIHT32 training grant HL076122 fellowship to SRB.

\section{Compliance with ethical standards}

Conflict of interest Vikas Anathy currently have awarded patents United States Patent No. 8,679,811, "Treatments Involving Glutaredoxins and Similar Agents", and United States Patent Nos, 9,907,828 and $10,688,150$ "Treatments of oxidative stress conditions". Vikas Anathy have received consultation fee and contract grants from Celdara Medical, LLC, NH.

\section{References}

Abrisch RG, Gumbin SC, Wisniewski BT, Lackner LL, Voeltz GK (2020) Fission and fusion machineries converge at ER contact sites to regulate mitochondrial morphology. J Cell Biol 219:e201911122. https://doi.org/10.1083/jcb.201911122

Aguilera-Aguirre L, Bacsi A, Saavedra-Molina A, Kurosky A, Sur S, Boldogh I (2009) Mitochondrial dysfunction increases allergic airway inflammation. J Immunol 183:5379-5387. https://doi. org/10.4049/jimmunol.0900228

Anderson DJ, Hetzer MW (2008) Reshaping of the endoplasmic reticulum limits the rate for nuclear envelope formation. J Cell Biol 182:911-924

Annesley SJ, Fisher PR (2019) Mitochondria in health and disease. Cells 8:680. https://doi.org/10.3390/cells8070680

Bhakta NR et al (2018) IFN-stimulated gene expression, type 2 inflammation, and endoplasmic reticulum stress in asthma. Am J Respir Crit Care Med 197:313-324. https://doi.org/10.1164/ rccm.201706-1070OC

Bian Q, Lu J, Zhang L, Chi Y, Li Y, Guo H (2017) Highly pathogenic avian influenza A virus $\mathrm{H} 5 \mathrm{~N} 1$ non-structural protein 1 is associated with apoptotic activation of the intrinsic mitochondrial pathway. Exp Ther Med 14:4041-4046. https://doi. org/10.3892/etm.2017.5056

Bílý T, Palus M, Eyer L, Elsterová J, Vancová M, Růžek D (2015) Electron tomography analysis of tick-borne encephalitis virus infection in human neurons. Sci Rep 5:1-15

Bliek AMVD, Shen Q, Kawajiri S (2013) Mechanisms of mitochondrial fission and fusion. Cold Spring Harb Perspect Biol 5:a011072. https://doi.org/10.1101/cshperspect.a011072

Boyapalle S, Wong T, Garay J, Teng M, Juan-Vergara HS, Mohapatra S, Mohapatra S (2012) Respiratory syncytial virus NS1 protein colocalizes with mitochondrial antiviral signaling protein MAVS following infection. PLoS ONE 7:e29386. https://doi. org/10.1371/journal.pone.0029386

Braakman I, Hebert DN (2013) Protein folding in the endoplasmic reticulum. Cold Spring Harb Perspect Biol 5:a013201. https:// doi.org/10.1101/cshperspect.a013201

Braidy N, Guillemin GJ, Mansour H, Chan-Ling T, Poljak A, Grant R (2011) Age related changes in NAD+ metabolism oxidative stress and sirt1 activity in wistar rats. PLoS ONE 6:e19194. https ://doi.org/10.1371/journal.pone.0019194

Bueno $\mathrm{M}$ et al (2015) PINK1 deficiency impairs mitochondrial homeostasis and promotes lung fibrosis. J Clin Invest 125:521-538. https://doi.org/10.1172/JCI74942

Burman A et al (2018) Localized hypoxia links ER stress to lung fibrosis through induction of C/EBP homologous protein. JCI Insight 3:e99543. https://doi.org/10.1172/jci.insight.99543
Chakrabarty S, Kabekkodu SP, Singh RP, Thangaraj K, Singh KK, Satyamoorthy K (2018) Mitochondria in Health and Disease. Mitochondrion 43:25-29. https://doi.org/10.1016/j. mito.2018.06.006

Chamberlain $\mathrm{N}$ et al (2019) Lung epithelial protein disulfide isomerase A3 (PDIA3) plays an important role in influenza infection, inflammation, and airway mechanics. Redox Biol 22:101129. https://doi.org/10.1016/j.redox.2019.101129

Chen G et al (2019) XBP1S regulates MUC5B in a promoter variantdependent pathway in idiopathic pulmonary fibrosis airway epithelia. Am J Respir Crit Care Med 200:220-234. https://doi. org/10.1164/rccm.201810-1972OC

Choi KJ et al (2014) Three-dimensional analysis of abnormal ultrastructural alteration in mitochondria of hippocampus of APP/ PSEN1 transgenic mouse. J Biosci 39:97-105. https://doi. org/10.1007/s12038-013-9406-8

Clapham DE (2007) Calcium signaling. Cell 131:1047-1058. https:// doi.org/10.1016/j.cell.2007.11.028

Craene J-OD et al (2006) Rtn1p Is Involved in Structuring the Cortical Endoplasmic Reticulum. Mol Biol Cell 17:3009-3020

Csordás G, Weaver D, Hajnóczky G (2018) ER-mitochondrial contact-ology: structure and signaling functions. Trends Cell Biol 28:523-540. https://doi.org/10.1016/j.tcb.2018.02.009

Dahl R, Larsen S, Dohlmann TL, Qvortrup K, Helge JW, Dela F, Prats C (2015) Three-dimensional reconstruction of the human skeletal muscle mitochondrial network as a tool to assess mitochondrial content and structural organization. Acta Physiol 213:145-155

Delbrel E et al (2018) HIF-1 $\alpha$ triggers ER stress and CHOP-mediated apoptosis in alveolar epithelial cells, a key event in pulmonary fibrosis. Sci Rep 8:17939. https://doi.org/10.1038/s41598-01836063-2

Delgado T et al (2019) Comparing 3D ultrastructure of presynaptic and postsynaptic mitochondria. Biol Open 8:bio044834

Domart M-C et al (2012) Acute manipulation of diacylglycerol reveals roles in nuclear envelope assembly \& endoplasmic reticulum morphology. PLoS ONE 7:e51150

Dudkina NV, Kouřil R, Peters K, Braun H-P, Boekema EJ (2010) Structure and function of mitochondrial supercomplexes. Biochim Biophys Acta 1797:664-670

Elgass KD, Smith EA, LeGros MA, Larabell CA, Ryan MT (2015) Analysis of ER-mitochondria contacts using correlative fluorescence microscopy and soft X-ray tomography of mammalian cells. J Cell Sci 128:2195-2804. https://doi.org/10.1242/ jcs. 169136

Eustaquioa T et al (2018) Electron microscopy techniques employed to explore mitochondrial defects in the developing rat brain following ketamine treatment. Exp Cell Res 37:164-170. https:// doi.org/10.1016/j.yexcr.2018.10.009

Fagone P, Jackowski S (2009) Membrane phospholipid synthesis and endoplasmic reticulum function. J Lipid Res 50:S311-S316. https://doi.org/10.1194/jlr.R800049-JLR200

Fakouri NB, Hansen TL, Desler C, Anugula S, Rasmussen LJ (2019) From powerhouse to perpetrator-mitochondria in health and disease. Biology (Basel) 8:35. https://doi.org/10.3390/biology802 0035

Filadi R, Theurey P, Pizzo P (2017) The endoplasmic reticulummitochondria coupling in health and disease: molecules, functions and significance. Cell Calcium 62:1-15. https://doi. org/10.1016/j.ceca.2017.01.003

Friedman JR, Voeltz GK (2011) The ER in 3-D: a multifunctional dynamic membrane network. Trends Cell Biol 21:709-717

Gillespie LK, Hoenen A, Morgan G, Mackenzie JM (2010) The endoplasmic reticulum provides the membrane platform for biogenesis of the flavivirus replication complex. J Virol 84:10438-10447 
Glancy B (2020) Visualizing mitochondrial form and function within the cell. Trends Mol Med 26:58-70

Glancy B et al (2017) Power grid protection of the muscle mitochondrial reticulum. Cell Rep 19:487-496

Graffe M, Zenisek D, Taraska JW (2015) A marginal band of microtubules transports and organizes mitochondria in retinal bipolar synaptic terminals. J Gen Physiol 146:109-117

Hassan IH et al (2012) Influenza A viral replication is blocked by inhibition of the inositol-requiring enzyme 1 (IRE1) stress pathway. J Biol Chem 287:4679-4689. https://doi.org/10.1074/jbc. M111.284695

Hassan I et al (2014) Inositol-requiring enzyme 1 inhibits respiratory syncytial virus replication. J Biol Chem 289:7537-7546. https ://doi.org/10.1074/jbc.M113.510594

Hawkins A et al (2015) A non-BRICHOS SFTPC Mutant (SP-CI73T) linked to interstitial lung disease promotes a late block in macroautophagy disrupting cellular proteostasis and mitophagy. Am J Physiol Lung Cell Mol Physiol 308:L33-L47. https://doi. org/10.1152/ajplung.00217.2014

Hebert DN, Garman SC, Molinari M (2005) The glycan code of the endoplasmic reticulum: asparagine-linked carbohydrates as protein maturation and quality-control tags. Trends Cell Biol 15:364-370. https://doi.org/10.1016/j.tcb.2005.05.007

Herst PM, Rowe MR, Carson GM, Berridge MV (2017) Functional mitochondria in health and disease. Front Endocrinol (Lausanne) 8:296. https://doi.org/10.3389/fendo.2017.00296

Hetz C (2012) The unfolded protein response: controlling cell fate decisions under ER Stress and beyond. Nat Rev Mol Cell Biol 13:89-102. https://doi.org/10.1038/nrm3270

Hetz C, Zhang K, Kaufman RJ (2020) Mechanisms, regulation and functions of the unfolded protein response. Nat Rev Mol Cell Biol. https://doi.org/10.1038/s41580-020-0250-z

Hetzer MW, Walther TC, Mattaj IW (2005) Pushing the envelope: structure, function, and dynamics of the nuclear periphery. Annu Rev Cell Dev Biol 21:347-380

Hoffman SM et al (2013) Endoplasmic reticulum stress mediates house dust mite-induced airway epithelial apoptosis and fibrosis. Respir Res 14:141. https://doi.org/10.1186/1465-9921-14-141

Hoffman SM et al (2016) Protein disulfide isomerase-endoplasmic reticulum resident protein 57 regulates allergen-induced airways inflammation, fibrosis, and hyperresponsiveness. J Allergy Clin Immunol 137:822-832. https://doi.org/10.1016/j. jaci.2015.08.018

$\mathrm{Hu} \mathrm{M}$ et al (2019) Respiratory syncytial virus co-opts host mitochondrial function to favour infectious virus production. Elife 8:e42448. https://doi.org/10.7554/eLife.42448

Ilacqua N, Sánchez-Álvarez M, Bachmann M, Costiniti V, Pozo MAD, Giacomello M (2017) Protein localization at mitochondria-ER contact sites in basal and stress conditions. Front Cell Dev Biol 5:107. https://doi.org/10.3389/fcell.2017.00107

Islam MN et al (2012) Mitochondrial transfer from bone-marrowderived stromal cells to pulmonary alveoli protects against acute lung injury. Nat Med 18:759-765. https://doi.org/10.1038/ nm. 2736

Joensuua M, Jokitalo E (2015) ER sheet-tubule balance is regulated by an array of actin filaments and microtubules. Exp Cell Rex 337:170-178

Joensuua M et al (2014) ER sheet persistence is coupled to myosin 1c-regulated dynamic actin filament arrays. Mol Biol Cell 25:1111-1126

Katoh M et al (2017) Polymorphic regulation of mitochondrial fission and fusion modifies phenotypes of microglia in neuroinflammation. Sci Rep 7:4942. https://doi.org/10.1038/s41598-017-05232 $-0$
Katzen J et al (2019) An SFTPC BRICHOS mutant links epithelial ER stress and spontaneous lung fibrosis. JCI Insight 4:e126125. https ://doi.org/10.1172/jci.insight.126125

Kim SR, Kim DI, Kim SH, Lee H, Lee KS, Cho SH, Lee YC (2014) NLRP3 inflammasome activation by mitochondrial ROS in bronchial epithelial cells is required for allergic inflammation. Cell Death Dis 5:e1498. https://doi.org/10.1038/cddis.2014.460

Kodiha M, Flamant E, Wang YM, Stochaj U (2018) Defining the shortterm effects of pharmacological 5-AMP activated kinase modulators on mitochondrial polarization, morphology and heterogeneity. PeerJ 6:e5469. https://doi.org/10.7717/peerj.5469

Konrádová DV, Čopová C, Suková B, Houštěk J (1985) Ultrastructure of the bronchial epithelium in three children with asthma. Pediatr Pulmonol 1:182-187. https://doi.org/10.1002/ppul.1950010403

Korfei M et al (2008) Epithelial endoplasmic reticulum stress and apoptosis in sporadic idiopathic pulmonary fibrosis. Am J Respir Crit Care Med 178:838-846. https://doi.org/10.1164/rccm.20080 2-313OC

Landeras-Bueno S, Fernández Y, Falcón A, Oliveros JC, Ortín J (2016) Chemical genomics identifies the PERK-mediated unfolded protein stress response as a cellular target for influenza virus inhibition. mBio 7:e00085-00116. https://doi.org/10.1128/mBio.00085 $-16$

Lawson WE et al (2008) Endoplasmic reticulum stress in alveolar epithelial cells is prominent in IPF: association with altered surfactant protein processing and herpesvirus infection. Am J Physiol Lung Cell Mol Physiol 294:L1119-L1126. https://doi. org/10.1152/ajplung.00382.2007

Lawson WE et al (2011) Endoplasmic reticulum stress enhances fibrotic remodeling in the lungs. Proc Natl Acad Sci USA 108:10562-10567. https://doi.org/10.1073/pnas.1107559108

Lee H, Yoon Y (2016) Mitochondrial fission and fusion. Biochem Soc Trans 44:1725-1735. https://doi.org/10.1042/BST20160129

Lee H-Y, Lee G-H, Kim H-R, Lee Y-C, Chae H-J (2020) Phosphatidylinositol 3-kinase- $\delta$ controls endoplasmic reticulum membrane fluidity and permeability in fungus-induced allergic inflammation in mice. Br J Pharmacol 177:1556-1567. https://doi.org/10.1111/ bph.14917

Li X, Qu B, He G, Cardona CJ, Song Y, Xing Z (2018) Critical role of HAX-1 in promoting avian influenza virus replication in lung epithelial cells. Mediat Inflamm 2018:3586132. https://doi. org/10.1155/2018/3586132

Liedmann S et al (2014) Viral suppressors of the RIG-I-mediated interferon response are pre-packaged in influenza virions. Nat Commun 5:5645. https://doi.org/10.1038/ncomms6645

Liesa M, Shirihai OS (2013) Mitochondrial dynamics in the regulation of nutrient utilization and energy expenditure. Cell Metab 17:491-506. https://doi.org/10.1016/j.cmet.2013.03.002

Lin X et al (2016) The influenza virus H5N1 infection can induce ROS production for viral replication and host cell death in A549 cells modulated by human $\mathrm{Cu} / \mathrm{Zn}$ superoxide dismutase (SOD1) overexpression. Viruses 8:13. https://doi.org/10.3390/v8010013

Lu L, Kirchhausen T (2012) Visualizing the high curvature regions of post-mitotic nascent nuclear envelope membrane. Commun Integr Biol 5:16-18

Mabalirajan U, Dinda AK, Kumar S, Roshan R, Gupta P, Sharma SK, Ghosh B (2008) Mitochondrial structural changes and dysfunction are associated with experimental allergic asthma. J Immunol 181:3540-3548. https://doi.org/10.4049/jimmunol.181.5.3540

Martino MB et al (2013) The ER stress transducer IRE1 $\beta$ is required for airway epithelial mucin production. Mucosal Immunol 6:639-654. https://doi.org/10.1038/mi.2012.105

Miyazaki N, Esaki M, Ogura T, Murata K (2014) Serial block-face scanning electron microscopy for three-dimensional analysis of 
morphological changes in mitochondria regulated by $\mathrm{Cdc} 48 \mathrm{p} /$ p97 ATPase. J Struct Biol 187:187-193

Morozov YM, Datta D, Paspalas CD, Arnsten AFT (2017) Ultrastructural evidence for impaired mitochondrial fission in the aged rhesus monkey dorsolateral prefrontal cortex. Neurobiol Aging 51:9-18. https://doi.org/10.1016/j.neurobiolaging.2016.12.001

Offerdahl DK, Dorward DW, Hansen BT, Bloom ME (2012) A threedimensional comparison of tick-borne flavivirus infection in mammalian and tick cell lines. PLoS ONE 7:e47912

Owens K, Park JH, Gourley S, Jones H, Kristian T (2015) Mitochondrial dynamics: cell-type and hippocampal region specific changes following global cerebral ischemia. J Bioenerg Biomembr 47:13-31. https://doi.org/10.1007/s10863-014-9575-7

Patel AS et al (2015) Epithelial cell mitochondrial dysfunction and PINK1 are induced by transforming growth factor-beta1 in pulmonary fibrosis. PLoS ONE 10:e0121246. https://doi. org/10.1371/journal.pone.0121246

Pérez-Berná AJ et al (2016) Structural changes in cells imaged by soft $\mathrm{X}$-ray cryo-tomography during hepatitis $\mathrm{C}$ virus infection. ACS Nano 10:6597-6611

Picard M, Taivassalo T, Gouspillou G, Hepple RT (2011) Mitochondria: isolation, structure and function. J Physiol 18:4413-4421

Popov V, Medvedev NI, Davies HA, Stewart MG (2005) Mitochondria form a filamentous reticular network in hippocampal dendrites but are present as discrete bodies in axons: a three-dimensional ultrastructural study. J Comp Neurol 492:50-65

Puhka M, Vihinen H, Joensuu M, Jokitalo E (2007) Endoplasmic reticulum remains continuous and undergoes sheet-to-tubule transformation during cell division in mammalian cells. J Cell Biol 179:895-909

Roberson EC et al (2012) Influenza induces endoplasmic reticulum stress, caspase-12-dependent apoptosis, and c-Jun N-terminal kinase-mediated transforming growth factor- $\beta$ release in lung epithelial cells. Am J Respir Cell Mol Biol 46:573-581. https:// doi.org/10.1165/rcmb.2010-0460OC

Roingeard P, Hourioux C, Blanchard E, Prensier G (2008) Hepatitis $\mathrm{C}$ virus budding at lipid droplet-associated $\mathrm{ER}$ membrane visualized by $3 \mathrm{D}$ electron microscopy. Histochem Cell Biol 130:561-566

Romanov RA et al (2019) Chemical synapses without synaptic vesicles: purinergic neurotransmission via a CALHM1 channel-mitochondrial signaling complex. Sci Signal 11:eaao1815

Rowland AA, Voeltz GK (2012) Endoplasmic reticulum-mitochondria contacts: function of the junction. Nat Rev Mol Cell Biol 13:607-625. https://doi.org/10.1038/nrm3440

Schwarz DS, Blower MD (2016) The endoplasmic reticulum: structure, function and response to cellular signaling. Cell Mol Life Sci 73:79-94

Shibata Y, Voeltz GK, Rapoport TA (2006) Rough sheets and smooth tubules. Cell 126:435-439. https://doi.org/10.1016/j. cell.2006.07.019

Siu K-L, Chan C-P, Kok K-H, Woo PC-Y, Jin D-Y (2014) Comparative analysis of the activation of unfolded protein response by spike proteins of severe acute respiratory syndrome coronavirus and human coronavirus HKU1. Cell Biosci 4:3. https://doi. org/10.1186/2045-3701-4-3

Song J, Chi M, Luo X, Song Q, Xia D, Shi B, Han J (2019) Non-structural protein $2 \mathrm{~B}$ of human rhinovirus 16 activates both PERK and ATF6 rather than IRE1 to trigger ER stress. Viruses 11:133. https://doi.org/10.3390/v11020133

Sosulski ML, Gongora R, Feghali-Bostwick C, Lasky JA, Sanchez CG (2017) Sirtuin 3 deregulation promotes pulmonary fibrosis. J Gerontol A Biol Sci Med Sci 72:595-602. https://doi. org/10.1093/gerona/glw151

Stefanik M et al (2018) Characterisation of Zika virus infection in primary human astrocytes. BMC Neurosci 19:1-8
Szabadkai G, Simoni AM, Chami M, Wieckowski MR, Youle RJ, Rizzuto R (2004) Drp-1-dependent division of the mitochondrial network blocks intraorganellar $\mathrm{Ca} 2+$ waves and protects against $\mathrm{Ca} 2+-$-mediated apoptosis. Mol Cell 16:59-68. https:// doi.org/10.1016/j.molcel.2004.09.026

Tanjore H, Cheng D-S, Degryse AL, Zoz DF, Abdolrasulnia R, Lawson WE, Blackwell TS (2015) Alveolar epithelial cells undergo epithelial-to-mesenchymal transition in response to endoplasmic reticulum stress. J Biol Chem 290:3277. https://doi.org/10.1074/ jbc.A110.181164

Thamsen M et al (2019) Small molecule inhibition of IRE1 $\alpha$ kinase/RNase has anti-fibrotic effects in the lung. PLoS ONE 14:e0209824. https://doi.org/10.1371/journal.pone.0209824

Torres-González E et al (2012) Role of endoplasmic reticulum stress in age-related susceptibility to lung fibrosis. Am J Respir Cell Mol Biol 46:748-756. https://doi.org/10.1165/rcmb.2011-0224OC

Ulloa $\mathrm{G}$ et al (2019) Lipid species affect morphology of endoplasmic reticulum: a sea urchin oocyte model of reversible manipulation. J Lipid Res 60:1880-1891

Vincent AE et al (2016) The spectrum of mitochondrial ultrastructural defects in mitochondrial myopathy. Sci Rep 6:30610. https://doi. org/10.1038/srep30610

Voeltz GK, Rolls MM, Rapoport TA (2002) Structural organization of the endoplasmic reticulum. EMBO Rep 3:944-950. https://doi. org/10.1093/embo-reports/kvf202

West M, Zurek N, Hoenger A, Voeltz GK (2011a) A 3D analysis of yeast ER structure reveals how ER domains are organized by membrane curvature. J Cell Biol 193:333-346

West M, Zurek N, Hoenger A, Voeltz GK (2011b) A 3D analysis of yeast ER structure reveals how ER domains are organized by membrane curvature. J Cell Biol 193:333-346. https://doi. org $/ 10.1083 /$ jcb. 201011039

Westrate LM, Lee JE, Prinz WA, Voeltz GK (2015) Form follows function: the importance of endoplasmic reticulum shape. Annu Rev Biochem 84:791-811

Wieckowski MR, Szabadkai G, Wasilewski M, Pinton P, Duszyński J, Rizzuto R (2006) Overexpression of adenine nucleotide translocase reduces $\mathrm{Ca} 2+$ signal transmission between the ER and mitochondria. Biochem Biophys Res Commun 348:393-399. https://doi.org/10.1016/j.bbrc.2006.07.072

Xing $\mathrm{Z}$ et al (2011) Host immune and apoptotic responses to avian influenza virus H9N2 in human tracheobronchial epithelial cells. Am J Respir Cell Mol Biol 44:24-33. https://doi.org/10.1165/ rcmb.2009-01200C

$\mathrm{Xu} \mathrm{W}$ et al (2016) Increased mitochondrial arginine metabolism supports bioenergetics in asthma. J Clin Invest 126:2465-2481. https ://doi.org/10.1172/JCI82925

Yamaguchi J et al (2012) Comprehensive application of an mtDsRed2$\mathrm{Tg}$ mouse strain for mitochondrial imaging. Transgenic Res 21:439-447. https://doi.org/10.1007/s11248-011-9539-1

Yoon YH et al (2020) Altered mitochondrial functions and morphologies in epithelial cells are associated with pathogenesis of chronic rhinosinusitis with nasal polyps. Allergy Asthma Immunol Res 12:653-668. https://doi.org/10.4168/aair.2020.12.4.653

Yu JH et al (2019) Coenzyme Q10 alleviates tacrolimus-induced mitochondrial dysfunction in kidney. FASEB J 33:12288-12298. https://doi.org/10.1096/fj.201900386RR

Yuan X et al (2018) Allergy immunotherapy restores airway epithelial barrier dysfunction through suppressing IL-25 -induced endoplasmic reticulum stress in asthma. Sci Rep 8:7950. https://doi. org/10.1038/s41598-018-26221-x

Zheng Q, Tong M, Ou B, Liu C, Hu C, Yang Y (2019) Isorhamnetin protects against bleomycin-induced pulmonary fibrosis by inhibiting endoplasmic reticulum stress and epithelial-mesenchymal transition. Int J Mol Med 43:117-126. https://doi.org/10.3892/ ijmm.2018.3965 
Zhong Q et al (2011) Role of endoplasmic reticulum stress in epithelial-mesenchymal transition of alveolar epithelial cells: effects of misfolded surfactant protein. Am J Respir Cell Mol Biol 45:498 509. https://doi.org/10.1165/rcmb.2010-0347OC
Publisher's Note Springer Nature remains neutral with regard to jurisdictional claims in published maps and institutional affiliations. 